Available online at http://jnfa.mathres.org
MATHRES Journal of Nonlinear Functional Analysis
A

\title{
INFINITELY MANY FAST HOMOCLINIC ORBITS FOR A CLASS OF SUPERQUADRATIC DAMPED VIBRATION SYSTEMS
}

\author{
MOHSEN TIMOUMI \\ Department of Mathematics, Faculty of Sciences, Monastir University, Tunisia
}

\begin{abstract}
In this paper, we consider the following damped vibration system $\ddot{u}(t)+q(t) \dot{u}(t)-L(t) u(t)+$ $\nabla W(t, u(t))=0, \forall t \in \mathbb{R}$, where $q \in C(\mathbb{R}, \mathbb{R}), L \in C\left(\mathbb{R}, \mathbb{R}^{N^{2}}\right)$ is a symmetric matrix valued function and $W(t, x) \in C^{1}\left(\mathbb{R} \times \mathbb{R}^{N}, \mathbb{R}\right)$. We prove the existence of infinitely many fast homoclinic solutions for the system when $Q(t)=\int_{0}^{t} q(s) d s \rightarrow+\infty$ as $|t| \rightarrow \infty, L$ is neither coercive nor uniformly positive definite and $W(t, x)$ is superquadratic at infinity in the second variable but does not satisfy the well-known superquadratic growth conditions like the Ambrosetti-Rabinowitz or the Fei's conditions.

Keywords. Damped vibration systems; Fast homoclinic solutions; Variational methods; Variant Fountain Theorem.
\end{abstract}

\section{INTRODUCTION}

In this paper, we consider the following damped vibration system

$$
\ddot{u}(t)+q(t) \dot{u}(t)-L(t) u(t)+\nabla W(t, u(t))=0, \forall t \in \mathbb{R}
$$

where $q: \mathbb{R} \rightarrow \mathbb{R}$ is a continuous function, $L \in C\left(\mathbb{R}, \mathbb{R}^{N^{2}}\right)$ is a symmetric matrix-valued function and $W: \mathbb{R} \times \mathbb{R}^{N} \rightarrow \mathbb{R}$ is a continuous function, differentiable in the second variable with continuous derivative $\nabla W(t, x)=\frac{\partial W}{\partial x}(t, x)$. If $q(t)=0$ for all $t \in \mathbb{R}$, then $(\mathscr{D} \mathscr{V})$ is just the following second order Hamiltonian system

$$
\ddot{u}(t)-L(t) u(t)+\nabla W(t, u(t))=0, \forall t \in \mathbb{R} .
$$

As a special case of dynamical systems, Hamiltonian systems play an important role in practical problems concerning relativistic mechanics, gaz dynamics, nuclear physics, fluid mechanics and others. A solution $u$ of $(\mathscr{H} \mathscr{S})$ is said to be homoclinic (to 0$)$ if $u(t) \rightarrow 0$ and $\dot{u}(t) \rightarrow 0$ as $|t| \rightarrow \infty$. $u$ is nontrivial if $u \neq 0$. With the aids of variational methods and critical point theory, the existence and the multiplicity of homoclinic orbits for $(\mathscr{H} \mathscr{S})$ have been extensively investigated recently. Let us briefly comment some known results on homoclinic solutions for

E-mail address: m_timoumi@yahoo.com.

Received August 13, 2019; Accepted February 11, 2020.

(C)2020 Journal of Nonlinear Functional Analysis 
$(\mathscr{H} \mathscr{S})$. Under the assumptions that $L(t)$ and $W(t, x)$ are independent of $t$ or $T$-periodic in $t$, the existence of homoclinic solutions for Hamiltonian system $(\mathscr{H} \mathscr{S})$ was extensively studied, see, for example, $[1,2,3,4,5,6]$. In this case, the existence of homoclinic solutions can be obtained by taking the limit of $2 k T$-periodic solutions of approximating problems. The periodic assumptions are very important in the study of homoclinic orbits for $(\mathscr{H} \mathscr{S})$ since the periodicity is used to control the lack of compactness due to the fact that $(\mathscr{H} \mathscr{S})$ is set on all $\mathbb{R}$. If $L(t)$ and $W(t, x)$ are neither autonomous nor periodic in $t$, the problem of the existence of homoclinic solutions of $(\mathscr{H} \mathscr{S})$ is quite different from the ones just described because of the lack of compactness of the Sobolev embedding. In 1991, Rabinowitz and Tanaka [7] introduced a type of coercive condition on the matix $L$ :

$$
l(t)=\inf _{|\xi|=1} L(t) \xi \cdot \xi \rightarrow+\infty \text { as }|t| \rightarrow \infty,
$$

where ". denotes the standard inner product in $\mathbb{R}^{N}$ and $|\cdot|$ is the induced norm. They established a compactness lemma under the nonperiodic case and obtained the existence of homoclinic orbits for system $(\mathscr{H} \mathscr{S})$ under the well-known Ambrosetti-Rabinowitz superquadratic growth (in short $(\mathscr{A} \mathscr{R})$ ) condition. Later, Ding [8] strengthened condition (1.1) by the following: there exists a constant $\alpha>0$ such that

$$
l(t)|t|^{-\alpha} \rightarrow+\infty \text { as }|t| \rightarrow \infty .
$$

Under condition (1.2) and some subquadratic conditions on $W(t, x)$, the author proved the existence and multiplicity of homoclinic orbits for the system $(\mathscr{H} \mathscr{S})$. Subsequently, conditions (1.1) and (1.2) have been extensively used, see, e.g., [9, 10, 11, 12, 13, 14, 15, 16, 17, 18, 19] and the references cited therein. However, it is worth pointing out that all of the assumptions on $L$ in the works mentioned above indicate that the smallest eigenvalue $l(t)=\inf _{|\xi|=1} L(t) \xi \cdot \xi$ of $L(t)$ is coercive, i.e., $l(t) \rightarrow+\infty$ as $|t| \rightarrow \infty$. The above condition does not seem to be natural. Recently, Tang [20] showed that it can be dropped. More precisely, he proved the existence of infinitely many homoclinic solutions for the Hamiltonian system $(\mathscr{H} \mathscr{S})$ when the potential $W$ satisfies some superquadratic conditions at infinity and the matrix-valued function $L$ is neither coercive nor uniformly positive definite. More precisely, $L$ is required to satisfy

$(L)$ The smallest eigenvalue $l(t)$ of $L(t)$ is bounded from below;

$\left(L_{0}\right)$ There exists a constant $r_{0}>0$ such that

$$
\lim _{|s| \rightarrow \infty} \operatorname{meas}\left(\{t \in] s-r_{0}, s+r_{0}\left[/ L(t)<b I_{N}\right\}\right)=0, \forall b>0,
$$

where meas denotes the Lebesgue's measure on $\mathbb{R}$ and $I_{N}$ is the $N \times N$ identity matrix. Here, for two $N \times N$ symmetric matrices $M_{1}$ and $M_{2}$, we say that $M_{1}<M_{2}$ if

$$
\min _{x \in \mathbb{R}^{N},|x|=1}\left(M_{1}-M_{2}\right) x \cdot x<0,
$$

and $M_{1} \geq M_{2}$ if $M_{1}<M_{2}$ does not hold. When $q \neq 0$, some authors have studied homoclinic orbits of $(\mathscr{D} \mathscr{V})$, see, e.g., $[21,22,23,24]$. In all these results, the matrix-valued function $L(t)$ is assumed to be coercive. Recently, Timoumi [23] obtained the existence of infinitely many fast homoclinic orbits for $(\mathscr{D} \mathscr{V})$ (see Definition 2.1), where $L(t)$ satisfies condition (1.2), and $W(t, x)$ satisfies the Fei's superquadratic growth (in short $(\mathscr{F})$ ) conditions. 
In this paper, motivated by [20,23], we will study the existence of infinitely many solutions for $(\mathscr{D} \mathscr{V})$, which are fast homoclinics, where $L$ is neither coercive nor uniformly positive definite and $W(t, x)$ satisfies some weak superquadratic conditions at infinity with respect to the second variable and does not check either $(\mathscr{A} \mathscr{R})$-condition or $(\mathscr{F})$-condition. Before presenting our main result, we make our hypotheses:

$(Q)\|q\|_{\infty}<\infty$ and $Q(t)=\int_{0}^{t} q(s) d s \rightarrow+\infty$ as $|t| \rightarrow \infty$;

$\left(L_{Q}\right)$ there exists a constant $r_{0}>0$ such that

$$
\lim _{|s| \rightarrow \infty} \operatorname{meas}_{Q}\left(\{t \in] s-r_{0}, s+r_{0}\left[/ L(t)<b I_{N}\right\}\right)=0, \forall b>0,
$$

where meas $Q$ denotes the Lebesgue's measure on $\mathbb{R}$ with density $e^{Q(t)}$, i.e. meas $Q(A)=\int_{A} e^{Q(t)} d t$; $\left(W_{1}\right) W(t, 0)=0$ and there exist constants $c>0$ and $v>2$ such that

$$
|\nabla W(t, x)| \leq c\left(|x|+|x|^{v-1}\right), \forall(t, x) \in \mathbb{R} \times \mathbb{R}^{N}
$$

$$
W(t, x) \geq 0, \forall(t, x) \in \mathbb{R} \times \mathbb{R}^{N} \text { and } \lim _{|x| \rightarrow \infty} \frac{W(t, x)}{|x|^{2}}=+\infty, \text { uniformly for } t \in \mathbb{R}
$$

$\left(W_{3}\right)$ there exists a constant $\sigma \geq 1$ such that

$$
\sigma \widehat{W}(t, x) \geq \widehat{W}(t, s x), \forall(s, t, x) \in[0,1] \times \mathbb{R} \times \mathbb{R}^{N},
$$

where $\widehat{W}(t, x)=\nabla W(t, x) \cdot x-2 W(t, x)$;

$$
W(t,-x)=W(t, x), \forall(t, x) \in \mathbb{R} \times \mathbb{R}^{N} .
$$

Our main result reads as follows.

Theorem 1.1. Assume that $(Q),(L),\left(L_{Q}\right)$ and $\left(W_{1}\right)-\left(W_{4}\right)$ are satisfied. Then system $(\mathscr{D} \mathscr{V})$ possesses a sequence of nontrival fast homoclinic orbits $\left(u_{k}\right)$ satisfying

$$
\frac{1}{2} \int_{\mathbb{R}} e^{Q(t)}\left[\left|\dot{u}_{k}\right|^{2}+L(t) u_{k} \cdot u_{k}\right] d t-\int_{\mathbb{R}} e^{Q(t)} W\left(t, u_{k}\right) d t \rightarrow+\infty \text { as } k \rightarrow \infty .
$$

Remark 1.2. a) In our result, $L(t)$ is unnecassarily required to be either uniformly positive definite or coercive. For example, let $L(t)=\left(t^{2} \cos ^{2} t-1\right) I_{N}$. Then $L$ satisfies $(L)$ and $\left(L_{Q}\right)$ but it does'nt satisfy neither (1.1) nor (1.2).

b) Let

$$
W(t, x)=a(t)\left[|x|^{2} \ln (e+|x|)-\frac{1}{2}|x|^{2}+e|x|-e^{2}(\ln (e+|x|)-1)\right],
$$

where $a$ is a continuous bounded function with a positive lower bound. Then an easy computation shows that $W$ satisfies $\left(W_{1}\right)-\left(W_{4}\right)$. However, $W$ does not satisfy the $(\mathscr{A} \mathscr{R})$-condition or the $(\mathscr{F})$-conditions. The remainder of this paper is organized as follows. In Section 2, some preliminary results are presented, in particular, a compact lemma. The third Section is devoted to the proof of our main result. 


\section{Preliminaries}

In order to introduce the concept of fast homoclinic solutions for $(\mathscr{D} \mathscr{V})$ conveniently, we firstly describe some properties of the weighted Sobolev space $E$ on which the certain variational functional associated with $(\mathscr{D} \mathscr{V})$ is defined and the fast homoclinic solutions of $(\mathscr{D} \mathscr{V})$ are the critical points of such functional. We shall use $L_{Q}^{2}(\mathbb{R})$ to denote the Hilbert space of measurable functions from $\mathbb{R}$ into $\mathbb{R}^{N}$ under the inner product

$$
<u, v>_{L_{Q}^{2}}=\int_{\mathbb{R}} e^{Q(t)} u(t) \cdot v(t) d t
$$

and the induced norm

$$
\|u\|_{L_{Q}^{2}}=\left(\int_{\mathbb{R}} e^{Q(t)}|u(t)|^{2} d t\right)^{\frac{1}{2}} .
$$

Similarly, $L_{Q}^{p}(\mathbb{R})(1 \leq p<\infty)$ denotes the Banach space of functions on $\mathbb{R}$ with values in $\mathbb{R}^{N}$ under the norm

$$
\|u\|_{L_{Q}^{p}}=\left(\int_{\mathbb{R}} e^{Q(t)}|u(t)|^{p} d t\right)^{\frac{1}{p}}
$$

and $L_{Q}^{\infty}(\mathbb{R})$ denotes the Banach space of functions on $\mathbb{R}$ with values in $\mathbb{R}^{N}$ under the norm

$$
\|u\|_{L_{Q}^{\infty}}=\operatorname{esssup}\left\{e^{\frac{Q(t)}{2}}|u(t)| / t \in \mathbb{R}\right\} .
$$

In this section, we assume that $L$ satisfies the following condition instead of condition $(L)$

$$
l(t)=\inf _{|\xi|=1} L(t) \xi \cdot \xi \geq 1, \forall t \in \mathbb{R}
$$

and we introduce the Hilbert space

$$
E=\left\{u \in H_{Q}^{1}(\mathbb{R}) / \int_{\mathbb{R}} e^{Q(t)} L(t) u(t) \cdot u(t) d t<\infty\right\}
$$

equipped with the following inner product

$$
<u, v>=\int_{\mathbb{R}} e^{Q(t)}[\dot{u}(t) \cdot \dot{v}(t)+L(t) u(t) \cdot v(t)] d t
$$

and the induced norm $\|u\|=<u, u>^{\frac{1}{2}}$. Here, $H_{Q}^{1}(\mathbb{R})$ denotes the Sobolev space

$$
H_{Q}^{1}(\mathbb{R})=\left\{u \in L_{Q}^{2}(\mathbb{R}) / \dot{u} \in L_{Q}^{2}(\mathbb{R})\right\}
$$

Evidently, $E$ is continuously embedded into $L_{Q}^{p}(\mathbb{R})$ for $2 \leq p \leq \infty$, i.e., for all $2 \leq p \leq \infty$, there exists a constant $\eta_{p}>0$ such that

$$
\|u\|_{L_{Q}^{p}} \leq \eta_{p}\|u\|, \forall u \in E .
$$

Definition 2.1. A solution $u$ of $(\mathscr{D} \mathscr{V})$ is called a fast homoclinic orbit if $u \in E$.

Lemma 2.2. Assume that $(Q),\left(L^{\prime}\right)$ and $\left(L_{Q}\right)$ are satisfied. Then $E$ is compactly embedded in $L_{Q}^{p}(\mathbb{R})$ for all $2 \leq p<\infty$. 
Proof. Let $\left(u_{n}\right) \subset E$ be a bounded sequence such that $u_{n} \rightarrow u$ in $E$. We shall show that $u_{n} \rightarrow u$ in $L_{Q}^{p}(\mathbb{R})$ for all $2 \leq p<\infty$.

Step 1. Consider the case $p=2$. Suppose, without loss of generality, that $u_{n} \rightarrow 0$ in $E$. For any $s \in \mathbb{R}$, we denote $I_{r_{0}}(s)$ the open interval in $\mathbb{R}$ centered at $s$ with radius $r_{0}$, i.e., $I_{r_{0}}(s)=$ ]$s-r_{0}, s+r_{0}$ [, where $r_{0}$ is the constant given in $\left(L_{Q}\right)$. Let $\left(s_{i}\right)_{i \in \mathbb{N}} \subset \mathbb{R}$ be a sequence of points such that $\mathbb{R}=\cup_{i=1}^{\infty} I_{r_{0}}\left(s_{i}\right)$ and each $t \in \mathbb{R}$ is contained in at most two such intervals. For any $r>0$ and $b>0$, let

$$
\begin{aligned}
& C(r, b)=\{t \in \mathbb{R} \backslash]-r, r\left[/ L(t) \geq b I_{N}\right\}, \\
& D(r, b)=\{t \in \mathbb{R} \backslash]-r, r\left[/ L(t)<b I_{N}\right\} .
\end{aligned}
$$

Choosing $b_{\varepsilon}>\frac{4}{\varepsilon} \sup _{n \in \mathbb{N}}\left\|u_{n}\right\|^{2}$, we have

$$
\begin{aligned}
\int_{C\left(r, b_{\varepsilon}\right)} e^{Q(t)}\left|u_{n}\right|^{2} d t & \leq \frac{1}{b_{\varepsilon}} \int_{C\left(r, b_{\varepsilon}\right)} e^{Q(t)} L(t) u_{n}(t) \cdot u_{n}(t) d t \\
& \leq \frac{1}{b_{\varepsilon}} \int_{\mathbb{R}} e^{Q(t)} L(t) u_{n}(t) \cdot u_{n}(t) d t \leq \frac{1}{b_{\varepsilon}}\left\|u_{n}\right\|^{2}<\frac{\varepsilon}{4}
\end{aligned}
$$

Now, we have

$$
\begin{aligned}
\int_{D\left(r, b_{\varepsilon}\right)} e^{Q(t)}\left|u_{n}\right|^{2} d t & \leq \sum_{i=1}^{\infty} \int_{D\left(r, b_{\varepsilon}\right) \cap I_{r_{0}}\left(s_{i}\right)} e^{Q(t)}\left|u_{n}\right|^{2} d t \\
& \leq \sum_{i=1}^{\infty}\left(\int_{D\left(r, b_{\varepsilon}\right) \cap I_{r_{0}}\left(s_{i}\right)} e^{Q(t)}\left|u_{n}\right|^{4} d t\right)^{\frac{1}{2}\left[\operatorname{meas}_{Q}\left(D\left(r, b_{\varepsilon}\right) \cap I_{r_{0}}\left(s_{i}\right)\right)\right]^{\frac{1}{2}}} \\
& \leq a_{r} \sum_{i=1}^{\infty}\left(\int_{I_{r_{0}}\left(s_{i}\right)} e^{Q(t)}\left|u_{n}\right|^{4} d t\right)^{\frac{1}{2}}
\end{aligned}
$$

where $a_{r}=\sup _{i \in \mathbb{N}}\left[\operatorname{meas}_{Q}\left(D\left(r, b_{\varepsilon}\right) \cap I_{r_{0}}\left(s_{i}\right)\right)\right]^{\frac{1}{2}}$. By inequality (2.1), we have

$$
\begin{aligned}
\left(\int_{I_{r_{0}}\left(s_{i}\right)} e^{Q(t)}\left|u_{n}\right|^{4} d t\right)^{\frac{1}{4}} & =\left(\int_{\mathbb{R}} e^{Q(t)}\left|\chi_{\mid I_{r_{0}}\left(s_{i}\right)} u_{n}\right|^{4} d t\right)^{\frac{1}{4}} \\
& \leq \eta_{4}\left\|\chi_{\mid I_{r_{0}}\left(s_{i}\right)} u_{n}\right\| \leq \eta_{4}\left\|u_{n}\right\|
\end{aligned}
$$

Hence

$$
\begin{aligned}
\int_{D\left(r, b_{\varepsilon}\right)} e^{Q(t)}\left|u_{n}\right|^{2} d t & \leq \eta_{4}^{2} a_{r} \sum_{i=1}^{\infty}\left\|\chi_{\mid I_{r_{0}}\left(s_{i}\right)} u_{n}\right\|^{2} \\
& =\eta_{4}^{2} a_{r} \sum_{i=1}^{\infty} \int_{I_{r_{0}}\left(s_{i}\right)} e^{Q(t)}\left[\left|\dot{u}_{n}\right|^{2}+L(t) u_{n}(t) \cdot u_{n}(t)\right] d t \\
& \leq 2 \eta_{4}^{2} a_{r} \sup _{n \in \mathbb{N}}\left\|u_{n}\right\|^{2} .
\end{aligned}
$$

By an easy computation, we show that $a_{r} \rightarrow 0$ as $r \rightarrow \infty$. Therefore there exists $r_{\varepsilon}>0$ such that

$$
\int_{D\left(r_{\varepsilon}, b_{\varepsilon}\right)} e^{Q(t)}\left|u_{n}\right|^{2} d t<\frac{\varepsilon}{4}
$$


which together with (2.2) implies

$$
\begin{aligned}
\int_{\mathbb{R} \backslash]-r_{\varepsilon}, r_{\varepsilon}[} e^{Q(t)}\left|u_{n}\right|^{2} d t & =\int_{C\left(r_{\varepsilon}, b_{\varepsilon}\right)} e^{Q(t)}\left|u_{n}\right|^{2} d t+\int_{D\left(r_{\varepsilon}, b_{\varepsilon}\right)} e^{Q(t)}\left|u_{n}\right|^{2} d t \\
& <\frac{\varepsilon}{2} .
\end{aligned}
$$

By Sobolev's embedding theorem, we have that $u_{k} \rightarrow 0$ uniformly on $\left[-r_{\varepsilon}, r_{\varepsilon}\right]$. Then there exists $k_{0}>0$ such that

$$
\int_{\left[-r_{\varepsilon}, r_{\varepsilon}\right]} e^{Q(t)}\left|u_{k}\right|^{2} d t<\frac{\varepsilon}{2}, \forall k \geq k_{0}
$$

Combining (2.4) with (2.5), by the arbitrary of $\varepsilon$, we can obtain that $u_{k} \rightarrow 0$ in $L_{Q}^{2}(\mathbb{R})$.

Step 2. Case: $2<p<\infty$. By [13, Lemma 2.3], for any $v \in H^{1}(\mathbb{R})$, we have

$$
\|v\|_{L^{\infty}} \leq \frac{\sqrt{2}}{2}\left[\int_{\mathbb{R}}\left(|\dot{v}|^{2}+|v|^{2}\right) d t\right]^{\frac{1}{2}}
$$

Let $u \in E$ and set $v(t)=e^{\frac{Q(t)}{2}} u(t)$. By $\left(L^{\prime}\right)$, one has

$$
\begin{aligned}
\int_{\mathbb{R}}\left(|\dot{v}|^{2}+|v|^{2}\right) d t & =\int_{\mathbb{R}} e^{Q(t)}\left(\left|\dot{u}+\frac{q}{2} u\right|^{2}+|u|^{2}\right) d t \\
& \leq \int_{\mathbb{R}} e^{Q(t)}\left[2|\dot{u}|^{2}+\left(1+\frac{\|q\|_{\infty}^{2}}{2}\right)|u|^{2}\right] d t \\
& \leq \int_{\mathbb{R}} e^{Q(t)}\left[2|\dot{u}|^{2}+\left(1+\frac{\|q\|_{\infty}^{2}}{2}\right) L(t) u(t) \cdot u(t)\right] d t \\
& \leq c^{2}(q)\|u\|^{2},
\end{aligned}
$$

where $c^{2}(q)=\max \left\{2,\left(1+\frac{\|q\|_{\infty}^{2}}{2}\right)\right\}$. Combining (2.6) and (2.7) yields

$$
\|u\|_{L_{Q}^{\infty}} \leq \frac{\sqrt{2}}{2} c(q)\|u\|
$$

Hence

$$
\begin{aligned}
\left(\int_{\mathbb{R}} e^{Q(t)}|u|^{p} d t\right)^{\frac{1}{p}} & =\left(\int_{\mathbb{R}} e^{Q(t)}|u|^{2}|u|^{p-2} d t\right)^{\frac{1}{p}} \\
& \leq\|u\|_{L^{\infty}}^{\frac{p-2}{2}}\|u\|_{L_{Q}^{2}}^{\frac{2}{p}} \\
& \leq\left(\frac{1}{m_{0}}\right)^{\frac{p-2}{2 p}}\|u\|_{L_{Q}^{\frac{p-2}{p}}}^{\frac{\sqrt{\infty}}{2}}\|u\|_{L_{Q}^{2}}^{\frac{2}{p}} \\
& \leq\left(\frac{\sqrt{2}}{2 m_{0}} c^{2}(q)\right)^{\frac{p-2}{2 p}}\|u\|^{\frac{p-2}{p}}\|u\|_{L_{Q}^{2}}^{\frac{2}{p}},
\end{aligned}
$$

where $m_{0}=\min _{t \in \mathbb{R}} e^{Q(t)}$. By virtue of Step 1 and (2.9), we obtain $u_{n} \rightarrow 0$ in $L_{Q}^{p}(\mathbb{R})$. The proof is completed.

To study the critical points of the variational functional associated with $(\mathscr{D} \mathscr{V})$, we need the following Variant Fountain Theorem established by Zou [25]. 
Let $E$ be a Banach space with the norm $\|$.$\| and E=\overline{\oplus_{j \in \mathbb{N}} X_{j}}$ with $\operatorname{dim} X_{j}<\infty$ for any $j \in \mathbb{N}$. Set $Y_{k}=\oplus_{j=1}^{k} X_{j}$ and $Z_{k}=\overline{\oplus_{j=k}^{\infty} X_{j}}$. Consider a family of functionals $f_{\lambda} \in C^{1}(E, \mathbb{R})$ of the type

$$
f_{\lambda}(u)=A(u)-\lambda B(u), u \in E, \lambda \in[1,2] .
$$

Lemma 2.3. (Variant Fountain Theorem) [25]. Assume that the functionals $f_{\lambda}$ satisfy (a) $f_{\lambda}$ maps bounded sets into bounded sets for all $\lambda \in[1,2]$ and

$$
f_{\lambda}(-u)=f_{\lambda}(u) \text { for all }(\lambda, u) \in[1,2] \times E ;
$$

(b) $B(u) \geq 0$ for all $u \in E$ and $A(u) \rightarrow+\infty$ or $B(u) \rightarrow+\infty$ as $\|u\| \rightarrow \infty$;

(c) There exist $\rho_{k}>r_{k}>0$ such that for all $\lambda \in[1,2]$

$$
\alpha_{k}(\lambda)=\inf _{u \in Z_{k},\|u\|=r_{k}} f_{\lambda}(u)>\beta_{k}(\lambda)=\max _{u \in Y_{k},\|u\|=\rho_{k}} f_{\lambda}(u) .
$$

Then

$$
\alpha_{k}(\lambda) \leq \xi_{k}(\lambda)=\inf _{\gamma \in \Gamma_{k}} \max _{u \in B_{k}} f_{\lambda}(\gamma(u)), \forall \lambda \in[1,2]
$$

where

$$
B_{k}=\left\{u \in Y_{k} /\|u\| \leq \rho_{k}\right\} \text { and } \Gamma_{k}=\left\{\gamma \in C\left(B_{k}, E\right) / \gamma \text { is odd, } \gamma_{\mid \partial B_{k}}=i d\right\} \text {. }
$$

Moreover, for almost every $\lambda \in[1,2]$, there exists a sequence $\left(u_{m}^{k}(\lambda)\right)_{m \in \mathbb{N}}$ such that

$$
\sup _{m \in \mathbb{N}}\left\|u_{m}^{k}(\lambda)\right\|<\infty, f_{\lambda}^{\prime}\left(u_{m}^{k}(\lambda)\right) \rightarrow 0, f_{\lambda}\left(u_{m}^{k}(\lambda)\right) \rightarrow \xi_{k}(\lambda) \text { as } m \rightarrow \infty .
$$

\section{Proof of Theorem 1.1}

First, note that $(L)$ implies that there exists a constant $a_{0}>0$ such that $L(t)+2 a_{0} I_{N} \geq I_{N}$ for all $t \in \mathbb{R}$. Let $\widetilde{L}(t)=L(t)+2 a_{0} I_{N}$ and $\widetilde{W}(t, x)=W(t, x)+a_{0}|x|^{2}$. Consider the following system

$$
\ddot{u}(t)+q(t) \dot{u}(t)-\widetilde{L}(t) u(t)+\nabla \widetilde{W}(t, u(t))=0, \forall t \in \mathbb{R} .
$$

Then (3.1) is equivalent to $(\mathscr{D} \mathscr{V})$. Moreover, it is easy to check that the hypotheses $\left(W_{1}\right)-\left(W_{4}\right)$ still hold for $\widetilde{W}(t, x)$ provided that those hold for $W(t, x)$ and the function $\widetilde{L}$ satisfies $\left(L^{\prime}\right)$ and $\left(L_{Q}\right)$. Hence, in what follows, we always assume, without loss of generality, that $L$ satisfies $\left(L^{\prime}\right)$ and $\left(L_{Q}\right)$.

Consider the variational function $f$ associated to the system $(D V)$ :

$$
f(u)=\frac{1}{2} \int_{\mathbb{R}} e^{Q(t)}\left(|\dot{u}(t)|^{2}+L(t) u(t) \cdot u(t)\right) d t-\int_{\mathbb{R}} e^{Q(t)} W(t, u(t)) d t
$$

defined on the Hilbert space $E$ introduced in Section 2. Set

$$
g(u)=\int_{\mathbb{R}} e^{Q(t)} W(t, u) d t, u \in E .
$$

Lemma 3.1. Assume that $(Q),\left(L^{\prime}\right),\left(L_{Q}\right)$ and $\left(W_{1}\right)$ are satisfied. Then $g \in C^{1}(E, \mathbb{R})$ and $g^{\prime}$ : $E \rightarrow E^{*}$ is compact, and $f \in C^{1}(E, \mathbb{R})$. Moreover, for all $u, v \in E$,

$$
g^{\prime}(u) v=\int_{\mathbb{R}} e^{Q(t)} \nabla W(t, u) \cdot v d t
$$


and

$$
\begin{aligned}
f^{\prime}(u) v & =\int_{\mathbb{R}} e^{Q(t)}[\dot{u} \cdot \dot{v}+L(t) u \cdot v] d t-\int_{\mathbb{R}} e^{Q(t)} \nabla W(t, u) \cdot v d t \\
& =<u, v>-\int_{\mathbb{R}} e^{Q(t)} \nabla W(t, u) \cdot v d t .
\end{aligned}
$$

Proof. By $\left(W_{1}\right)$, for any $s \in[0,1]$ and $u, v \in E$, we have

$$
\begin{aligned}
|\nabla W(t, u+s v) v| & \leq c\left(|u+s v|+|u+s v|^{v-1}\right)|v| \\
& \leq c\left[|u|+|v|+2^{v-2}\left(|u|^{v-1}+|v|^{v-1}\right)\right]|v| \\
& \leq c 2^{v-2}\left[|u||v|+|v|^{2}+|u|^{v-1}|v|+|v|^{v}\right] .
\end{aligned}
$$

The Hölder's inequality implies

$$
\int_{\mathbb{R}} e^{Q(t)}\left(|u||v|+|u|^{v-1}|v|\right) d t \leq\|u\|_{L_{Q}^{2}}\|v\|_{L_{Q}^{2}}+\|u\|_{L_{Q}^{v}}^{v-1}\|v\|_{L_{Q}^{v}}
$$

Hence, by (3.4), (3.5), the Mean Value Theorem and Lebesgue's Dominated Convergence Theorem, we get, for all $u, v \in E$,

$$
\begin{aligned}
\lim _{s \rightarrow 0} \frac{g(u+s v)-g(u)}{s} & =\lim _{s \rightarrow 0} \int_{\mathbb{R}} \int_{0}^{1} e^{Q(t)} \nabla W(t, u+\theta s v) \cdot v d \theta d t \\
& =\int_{\mathbb{R}} e^{Q(t)} \nabla W(t, u) \cdot v d t \\
& =J(u, v) .
\end{aligned}
$$

Moreover, it follows from (2.1), $\left(W_{1}\right)$ and (3.5) that

$$
\begin{aligned}
|J(u, v)| & \leq \int_{\mathbb{R}} e^{Q(t)}|\nabla W(t, u)||v| d t \\
& \leq c \int_{\mathbb{R}} e^{Q(t)}\left(|u||v|+|u|^{v-1}|v|\right) d t \\
& \leq c\left(\|u\|_{L_{Q}^{2}}\|v\|_{L_{Q}^{2}}+\|u\|_{L_{Q}^{v-1}}^{v-1} \|_{L_{Q}^{2}}\right) \\
& \leq c\left(\eta_{2}^{2}\|u\|+\eta_{v}^{v}\|u\|^{v-1}\right)\|v\| .
\end{aligned}
$$

Therefore, $J(u,$.$) is linear and bounded, and J(u,$.$) is the Gâteaux derivative of g$ at $u$.

Next, we prove that $J(u,$.$) is weakly continuous in u$. To this end, we first claim that if $u_{n} \rightarrow u$ in $E$, then $\nabla W\left(t, u_{n}\right) \rightarrow \nabla W(t, u)$ in $L_{Q}^{2}(\mathbb{R})$. Arguing indirectly, by Lemma 2.2, we may assume that there exists a subsequence $\left(u_{n_{k}}\right)$ such that

$$
u_{n_{k}} \rightarrow u \text { in both } L_{Q}^{2}(\mathbb{R}) \text { and } L_{Q}^{2(v-1)}(\mathbb{R}) \text { and } u_{n_{k}} \rightarrow \text { u a.e. in } \mathbb{R} \text { as } k \rightarrow \infty
$$

and

$$
\int_{\mathbb{R}} e^{Q(t)}\left|\nabla W\left(t, u_{n_{k}}\right)-\nabla W(t, u)\right|^{2} d t \geq \varepsilon_{0}, \forall k \in \mathbb{N}
$$

for some positive constant $\varepsilon_{0}$. By (3.7) and up to a subsequence if necessary, we can assume that $\sum_{k=1}^{\infty}\left\|u_{n_{k}}-u\right\|_{L_{Q}^{2}}<\infty$ and $\sum_{k=1}^{\infty}\left\|u_{n_{k}}-u\right\|_{L_{Q}^{2(v-1)}}<\infty$. Let $w(t)=\sum_{k=1}^{\infty}\left|u_{n_{k}}(t)-u(t)\right|$ for all 
$t \in \mathbb{R}$. Then $w \in L_{Q}^{2}(\mathbb{R}) \cap L_{Q}^{2(v-1)}(\mathbb{R})$. By $\left(W_{1}\right)$, there holds for all $k \in \mathbb{N}$ and $t \in \mathbb{R}$

$$
\begin{aligned}
& \left|\nabla W\left(t, u_{n_{k}}\right)-\nabla W(t, u)\right|^{2} \\
& \leq\left(\left|\nabla W\left(t, u_{n_{k}}\right)\right|+|\nabla W(t, u)|\right)^{2} \\
& \leq 2\left(\left|\nabla W\left(t, u_{n_{k}}\right)\right|^{2}+|\nabla W(t, u)|^{2}\right) \\
& \leq 2 c^{2}\left[\left(\left|u_{n_{k}}\right|+\left|u_{n_{k}}\right|^{v-1}\right)^{2}+\left(|u|+|u|^{v-1}\right)^{2}\right] \\
& \leq 2^{2} c^{2}\left[\left|u_{n_{k}}\right|^{2}+\left|u_{n_{k}}\right|^{2(v-1)}+|u|^{2}+|u|^{2(v-1)}\right] \\
& \leq 2^{2} c^{2}\left[\left(\left|u_{n_{k}}-u\right|+|u|\right)^{2}+\left(\left|u_{n_{k}}-u\right|+|u|\right)^{2(v-1)}+|u|^{2}+|u|^{2(v-1)}\right] \\
& \leq 2^{2} c^{2}\left[2\left(\left|u_{n_{k}}-u\right|^{2}+|u|^{2}\right)+2^{2 v-3}\left(\left|u_{n_{k}}-u\right|^{2(v-1)}+|u|^{2(v-1)}\right)+|u|^{2}+|u|^{2(v-1)}\right] \\
& \leq c_{1}\left(|w|^{2}+|u|^{2}+|w|^{2(v-1)}+|u|^{2(v-1)}\right),
\end{aligned}
$$

where $c_{1}$ is a positive constant. Combining this with (3.7), Lebesgue's Dominated Convergence Theorem implies

$$
\lim _{k \rightarrow \infty} \int_{\mathbb{R}} e^{Q(t)}\left|\nabla W\left(t, u_{n_{k}}\right)-\nabla W(t, u)\right|^{2} d t=0
$$

which contradicts to (3.8). Hence the claim above is true.

Now, we suppose $u_{n} \rightarrow u$ in $E$. Then $\nabla W\left(t, u_{n}\right) \rightarrow \nabla W(t, u)$ in $L_{Q}^{2}(\mathbb{R})$. By Hölder's inequality and (2.1), we have

$$
\begin{aligned}
\left\|J\left(u_{n}, .\right)-J(u, .)\right\|_{E^{*}} & =\sup _{\|v\|=1} \int_{\mathbb{R}} e^{Q(t)}\left(\nabla W\left(t, u_{n}\right)-\nabla W(t, u)\right) \cdot v d t \\
& \leq \eta_{2}\left(\int_{\mathbb{R}} e^{Q(t)}\left|\nabla W\left(t, u_{n}\right)-\nabla W(t, u)\right|^{2} d t\right)^{\frac{1}{2}} \rightarrow 0 \text { as } n \rightarrow \infty .
\end{aligned}
$$

This means that $u \longmapsto J(u,$.$) is weakly continuous and then it is continuous in E$. Therefore $g \in C^{1}(E, \mathbb{R})$ and (3.2) is verified. Furthermore, $g^{\prime}$ is compact by the weak continuity of $g^{\prime}$ since $E$ is reflexive. Due to the form of $f$, (3.3) is also verified and $f \in C^{1}(E, \mathbb{R})$.

Finally, let $u \in E$ be a critical point of $f$. A standard argument shows that $u \in C^{2}(\mathbb{R})$ and satisfies equation $(\mathscr{D} \mathscr{V})$. The proof is completed.

In order to apply the Variant Fountain Theorem to prove our main result, we choose an orthonormal basis $\left(e_{n}\right)_{n \in \mathbb{N}}$ of $E$ and let $X_{j}=\operatorname{span}\left\{e_{j}\right\}$ for all $j \in \mathbb{N}$. Define $Y_{k}$ and $Z_{k}$ by

$$
Y_{k}=\oplus_{j=1}^{k} X_{j}, Z_{k}=\overline{\oplus_{j=k}^{\infty} X_{j}}
$$

and the functionals $A, B$ and $f_{\lambda}$ on our working space $E$ by

$$
A(u)=\frac{1}{2}\|u\|^{2}, B(u)=g(u)=\int_{\mathbb{R}} e^{Q(t)} W(t, u) d t, f_{\lambda}(u)=A(u)-\lambda B(u),
$$

for all $(\lambda, u) \in[1,2] \times E$.

Assumption $\left(W_{1}\right)$ and property (2.1) imply that $f_{\lambda}$ maps bounded sets into bounded sets uniformly for $\lambda \in[1,2]$. Note that $W(t,-x)=W(t, x)$. So $f_{\lambda}(-u)=f_{\lambda}(u)$ for all $(\lambda, u) \in[1,2] \times E$. Thus condition a) of Lemma 2.3 holds. Since $W(t, x) \geq 0$ for all $(t, x) \in \mathbb{R} \times \mathbb{R}^{N}$, it is clear that condition $b$ ) is also satisfied. To verify condition $c)$, we need to establish the three following lemmas. 
Lemma 3.2. Suppose that $(Q),\left(L^{\prime}\right)$ and $\left(L_{Q}\right)$ hold. Then, for any $p \in[2, \infty]$,

$$
l_{p}(k)=\sup _{u \in Z_{k},\|u\|=1}\|u\|_{L_{Q}^{p}} \rightarrow 0 \text { as } k \rightarrow \infty .
$$

Proof. It is clear that $0<l_{p}(k+1) \leq l_{p}(k)$ so that $l_{p}(k) \rightarrow \bar{l}_{p}$ as $k \rightarrow \infty$. For every $k \geq 1$, there exists $u_{k} \in Z_{k}$ such that $\left\|u_{k}\right\|=1$ and $\left\|u_{k}\right\|_{L_{Q}^{p}}>\frac{1}{2} l_{p}(k)$. For any $v \in E$, let $v=\sum_{i=1}^{\infty} v_{i} e_{i}$. By the Cauchy-Schartz inequality, one has

$$
\begin{aligned}
\left|<u_{k}, v>\right| & =\left|<u_{k}, \sum_{i=1}^{\infty} v_{i} e_{i}>\right| \\
& =\left|<u_{k}, \sum_{i=k+1}^{\infty} v_{i} e_{i}>\right| \\
& \leq\left\|u_{k}\right\|\left\|\sum_{i=k+1}^{\infty} v_{i} e_{i}\right\| \\
& \leq \sum_{i=k+1}^{\infty}\left|v_{i}\right|\left\|e_{i}\right\| \rightarrow 0 \text { as } k \rightarrow \infty,
\end{aligned}
$$

which implies that $u_{k} \rightarrow 0$. Without loss of generality, Lemma 2.2 implies that $u_{k} \rightarrow 0$ in $L_{Q}^{p}(\mathbb{R})$. Thus we have proved that $\bar{l}_{p}(k)=0$. The proof is completed.

Lemma 3.3. Assume that $(Q),\left(L^{\prime}\right),\left(L_{Q}\right),\left(W_{1}\right)$ and $\left(W_{2}\right)$ are satisfied. Then there exist a positive integer $k_{0}$ and a sequence $r_{k} \rightarrow+\infty$ as $k \rightarrow \infty$ such that

$$
\alpha_{k}(\lambda)=\inf _{u \in Z_{k},\|u\|=r_{k}} f_{\lambda}(u)>0, \forall k \geq k_{0} .
$$

Proof. From $\left(W_{1}\right)$ and the fact that $W(t, x) \geq 0$, we have

$$
\begin{aligned}
f_{\lambda}(u) & \geq \frac{1}{2}\|u\|^{2}-2 \int_{\mathbb{R}} e^{Q(t)} W(t, u) d t \\
& \geq \frac{1}{2}\|u\|^{2}-2 c\left(\|u\|_{L_{Q}^{2}}^{2}+\|u\|_{L_{Q}^{v}}^{v}\right), \forall(\lambda, u) \in[1,2] \times E .
\end{aligned}
$$

Combining (3.9) and (3.11) yields

$$
f_{\lambda}(u) \geq \frac{1}{2}\|u\|^{2}-2 c l_{2}^{2}(k)\|u\|^{2}-2 c l_{v}^{v}(k)\|u\|^{v}, \forall(\lambda, u) \in[1,2] \times E .
$$

In view of (3.9), there exists an integer $k_{0}$ such that

$$
2 c l_{2}^{2}(k) \leq \frac{1}{4}, \forall k \geq k_{0} .
$$

For any $k \geq k_{0}$, let us define

$$
r_{k}=\left(16 c l_{v}^{v}(k)\right)^{\frac{1}{2-v}} .
$$

Since $v>2$, we have $r_{k} \rightarrow+\infty$ as $k \rightarrow \infty$. From (3.12), (3.13) and (3.14), we deduce that, for all $k \geq k_{0}$,

$$
\inf _{u \in Z_{k},\|u\|=r_{k}} f_{\lambda}(u) \geq \frac{1}{2} r_{k}^{2}-\frac{1}{4} r_{k}^{2}-\frac{1}{8} r_{k}^{2-v} r_{k}^{v}=\frac{1}{8} r_{k}^{2}>0,
$$

which completes the proof. 
Lemma 3.4. Assume that $(Q),\left(L^{\prime}\right),\left(L_{Q}\right),\left(W_{1}\right)$ and $\left(W_{2}\right)$ are satisfied. Then, for any $k \geq k_{0}$, there exists $\rho_{k}>r_{k}$ such that

$$
\beta_{k}(\lambda)=\max _{u \in Y_{k},\|u\|=\rho_{k}} f_{\lambda}(u)<0,
$$

where $k_{0}$ is the positive integer obtained in Lemma 3.3.

Proof. Firstly, we claim that for any finite-dimensional subspace $F \subset E$, there exists a constant $\varepsilon_{0}>0$ such that

$$
\operatorname{meas}_{Q}\left(\left\{t \in \mathbb{R} /|u(t)| \geq \varepsilon_{0}\|u\|\right\}\right) \geq \varepsilon_{0}, \forall u \in F \backslash\{0\} .
$$

In not, for any $n \in \mathbb{N}$, there exists $u_{n} \in F \backslash\{0\}$ such that

$$
\operatorname{meas}_{Q}\left(\left\{t \in \mathbb{R} /\left|u_{n}(t)\right| \geq \frac{1}{n}\left\|u_{n}\right\|\right\}\right)<\frac{1}{n} .
$$

Let $v_{n}=\frac{u_{n}}{\left\|u_{n}\right\|} \in F$. Then $\left\|v_{n}\right\|=1$ and

$$
\operatorname{meas}_{Q}\left(\left\{t \in \mathbb{R} /\left|v_{n}(t)\right| \geq \frac{1}{n}\right\}\right)<\frac{1}{n}, \forall n \in \mathbb{N} .
$$

Since $\mathrm{F}$ is finite-dimensional, then up to a subsequence if necessary, we may assume that $v_{n} \rightarrow v_{0}$ in $E$ for some $v_{0} \in F$. Evidently, $\left\|v_{0}\right\|=1$. Note that, since any two norms on $F$ are equivalent, we have

$$
\int_{\mathbb{R}} e^{Q(t)}\left|v_{n}-v_{0}\right| d t \rightarrow 0 \text { as } n \rightarrow \infty .
$$

The fact that $\left\|v_{0}\right\|=1$ implies $\left\|v_{0}\right\|_{L_{Q}^{\infty}}>0$. By the definition of $\|\cdot\|_{L_{Q}^{\infty}}$, there exists a constant $\delta_{0}>0$ such that

$$
\operatorname{meas}_{Q}\left(\left\{t \in \mathbb{R} /\left|v_{0}(t)\right| \geq \delta_{0}\right\}\right) \geq \delta_{0} .
$$

Otherwise, for each fixed $n \in \mathbb{N}$ and $m>n$, we have

$$
\begin{aligned}
\operatorname{meas}_{Q}\left(\left\{t \in \mathbb{R} /\left|v_{0}(t)\right| \geq \frac{1}{n}\right\}\right) & \leq \operatorname{meas}_{Q}\left(\left\{t \in \mathbb{R} /\left|v_{0}(t)\right| \geq \frac{1}{m}\right\}\right) \\
& \leq \frac{1}{m} .
\end{aligned}
$$

Letting $m \rightarrow \infty$, we obtain meas m $_{Q}\left(\left\{t \in \mathbb{R} /\left|v_{0}(t)\right| \geq \frac{1}{n}\right\}\right)=0$. Consequently

$$
\begin{aligned}
0 & \leq \text { meas }_{Q}\left(\left\{t \in \mathbb{R} /\left|v_{0}(t)\right| \neq 0\right\}\right) \\
& =\text { meas }_{Q}\left(\cup_{n=1}^{\infty}\left\{t \in \mathbb{R} /\left|v_{0}(t)\right| \geq \frac{1}{n}\right\}\right) \\
& \leq \sum_{n=1}^{\infty} \text { meas }_{Q}\left(\left\{t \in \mathbb{R} /\left|v_{0}(t)\right| \geq \frac{1}{n}\right\}\right)=0,
\end{aligned}
$$

which yields $v_{0}=0$ and contradicts $\left\|v_{0}\right\|=1$. Then (3.19) holds. For any $n \in \mathbb{N}$, let

$$
\Lambda_{n}=\left\{t \in \mathbb{R} /\left|v_{n}(t)\right|<\frac{1}{n}\right\}, \Lambda_{0}=\left\{t \in \mathbb{R} /\left|v_{0}(t)\right| \geq \delta_{0}\right\}
$$


Then, for $n$ large enough, we have from (3.17) and (3.19) that

$$
\begin{aligned}
\text { meas }_{Q}\left(\Lambda_{n} \cap \Lambda_{0}\right) & \geq \text { meas }_{Q}\left(\Lambda_{0}\right)-\text { meas }_{Q}\left(\Lambda_{n}^{c}\right) \\
& \geq \delta_{0}-\frac{1}{n} \\
& \geq \frac{\delta_{0}}{2} .
\end{aligned}
$$

Consequently, for $n$ large enough, there holds

$$
\begin{aligned}
\int_{\mathbb{R}} e^{Q(t)}\left|v_{n}-v_{0}\right| d t & \geq \int_{\Lambda_{n} \cap \Lambda_{0}} e^{Q(t)}\left|v_{n}-v_{0}\right| d t \\
& \geq \int_{\Lambda_{n} \cap \Lambda_{0}} e^{Q(t)}\left(\left|v_{0}\right|-\left|v_{n}\right|\right) d t \\
& \geq\left(\delta_{0}-\frac{1}{n}\right) \operatorname{meas}_{Q}\left(\Lambda_{n} \cap \Lambda_{0}\right) \\
& \geq \frac{\delta_{0}^{2}}{4}>0 .
\end{aligned}
$$

This contradicts to (3.18). Therefore (3.16) holds.

Now, note that, for any $k \in \mathbb{N}, Y_{k}$ is finite-dimensional. So there exists a constant $\varepsilon_{k}>0$ such that

$$
\operatorname{meas}_{Q}\left(\Lambda_{u}^{k}\right) \geq \varepsilon_{k}, \forall u \in Y_{k} \backslash\{0\}
$$

where

$$
\Lambda_{u}^{k}=\left\{t \in \mathbb{R} /|u(t)| \geq \varepsilon_{k}\|u\|\right\}
$$

for all $k \in \mathbb{N}$ and $u \in Y_{k} \backslash\{0\}$. By $\left(W_{2}\right)$, for any $k \in \mathbb{N}$, there exists a constant $R_{k}>0$ such that

$$
W(t, x) \geq \frac{|x|^{2}}{\varepsilon_{k}^{3}}, \forall t \in \mathbb{R} \text { and }|x| \geq R_{k} .
$$

Combining (3.20) with (3.21), for any $k \in \mathbb{N}$ and $\lambda \in[1,2]$, we have

$$
\begin{aligned}
f_{\lambda}(u) & \leq \frac{1}{2}\|u\|^{2}-\int_{\Lambda_{u}^{k}} e^{Q(t)} W(t, u) d t \\
& \leq \frac{1}{2}\|u\|^{2}-\int_{\Lambda_{u}^{k}} e^{Q(t)} \frac{|u|^{2}}{\varepsilon_{k}^{3}} d t \\
& \leq \frac{1}{2}\|u\|^{2}-\varepsilon_{k}^{2}\|u\|^{2} \operatorname{meas}_{Q}\left(\Lambda_{u}^{k}\right) \frac{1}{\varepsilon_{k}^{3}} \\
& \leq \frac{1}{2}\|u\|^{2}-\|u\|^{2} \\
& =-\frac{1}{2}\|u\|^{2}
\end{aligned}
$$

for all $u \in Y_{k}$ with $\|u\| \geq \frac{R_{k}}{\varepsilon_{k}}$. For any $k \geq k_{0}$, we choose $\rho_{k}>\max \left\{r_{k}, \frac{R_{k}}{\varepsilon_{k}}\right\}$. It follows from (3.22) that

$$
\rho_{k}(\lambda)=\max _{u \in Y_{k},\|u\|=\rho_{k}} f_{\lambda}(u) \leq-\frac{1}{2} \rho_{k}^{2} .
$$

The proof is now completed. 
Consequently, Lemmas 3.3,3.4 show that condition $c$ ) of Lemma 2.3 is satisfied for all $k \geq k_{0}$. From the above, all the conditions of Lemma 2.3 hold for all $k \geq k_{0}$. Therefore, for any $k \geq k_{0}$ and $\lambda \in[1,2]$, there exists a sequence $\left(u_{n}^{k}(\lambda)\right) \subset E$ such that

$$
\sup _{n \in \mathbb{N}}\left\|u_{n}^{k}(\lambda)\right\|<\infty, f_{\lambda}^{\prime}\left(u_{n}^{k}(\lambda)\right) \rightarrow 0 \text { and } f_{\lambda}\left(u_{n}^{k}(\lambda)\right) \rightarrow \xi_{k}(\lambda)
$$

as $n \rightarrow \infty$, where

$$
\xi_{k}(\lambda)=\inf _{\gamma \in \Gamma_{k}} \max _{u \in B_{k}} f_{\lambda}(u), \forall \lambda \in[1,2]
$$

with

$$
B_{k}=\left\{u \in Y_{k} /\|u\| \leq \rho_{k}\right\} \text { and } \Gamma_{k}=\left\{\gamma \in C\left(B_{k}, E\right) / \gamma \text { is odd, } \gamma_{\mid \partial B_{k}}=i d\right\} \text {. }
$$

From (3.15) and Lemma 2.3, we infer that

$$
\xi_{k}(\lambda) \in\left[\bar{\alpha}_{k}, \bar{\xi}_{k}\right], \forall k \geq k_{0} \text { and } \lambda \in[1,2],
$$

where $\bar{\xi}_{k}=\max _{u \in B_{k}} f_{1}(u)$ and $\bar{\alpha}_{k}=\frac{r_{k}^{2}}{8} \rightarrow \infty$ as $k \rightarrow \infty$.

$$
\sup _{m \in \mathbb{N}}\left\|u_{m}^{k}\left(\lambda_{n}\right)\right\|<\infty, f_{\lambda_{n}}^{\prime}\left(u_{m}^{k}\left(\lambda_{n}\right)\right) \rightarrow 0 \text { and } f_{\lambda_{n}}\left(u_{m}^{k}\left(\lambda_{n}\right)\right) \rightarrow \xi_{k}\left(\lambda_{n}\right) \text {, as } m \rightarrow \infty \text {. }
$$

Lemma 3.5. For any $n \in \mathbb{N}$ and $k \geq k_{0}$, there exists $u_{n}^{k} \in E$ such that

$$
\lim _{m \rightarrow \infty} u_{m}^{k}\left(\lambda_{n}\right)=u_{n}^{k} \text { in } E .
$$

Proof. Throughout this proof and for the sake of simplicity, we shall let $u_{m}=u_{m}^{k}\left(\lambda_{n}\right)$ for $m \in \mathbb{N}$. Without loss of generality, we may assume by (3.25) that

$$
u_{m} \rightarrow \text { us } m \rightarrow \infty
$$

for some $u \in E$. Using (3.3), we get

$$
\begin{aligned}
\left\|u_{m}-u\right\|^{2} & =f_{\lambda_{n}}^{\prime}\left(u_{m}\right)\left(u_{m}-u\right)-f_{\lambda_{n}}^{\prime}(u)\left(u_{m}-u\right) \\
& +\lambda_{n} \int_{\mathbb{R}} e^{Q(t)}\left(\nabla W\left(t, u_{m}\right)-\nabla W(t, u)\right) \cdot\left(u_{m}-u\right) d t .
\end{aligned}
$$

By (3.25), one has

$$
f_{\lambda_{n}}^{\prime}\left(u_{m}\right)\left(u_{m}-u\right) \rightarrow 0 \text { as } m \rightarrow \infty
$$

Moreover (3.27) implies

$$
f_{\lambda_{n}}^{\prime}(u)\left(u_{m}-u\right) \rightarrow 0 \text { as } m \rightarrow \infty
$$

Now, by (2.1) and Hölder's inequality, we have

$$
\begin{aligned}
& \left|\int_{\mathbb{R}} e^{Q(t)}\left(\nabla W\left(t, u_{m}\right)-\nabla W(t, u)\right) \cdot\left(u_{m}-u\right) d t\right| \\
& \leq\left(\int_{\mathbb{R}} e^{Q(t)}\left|\nabla W\left(t, u_{m}\right)-\nabla W(t, u)\right|^{2} d t\right)^{\frac{1}{2}}\left(\int_{\mathbb{R}} e^{Q(t)}\left|u_{m}-u\right|^{2} d t\right)^{\frac{1}{2}} \\
& \leq \eta_{2}\left(\int_{\mathbb{R}} e^{Q(t)}\left|\nabla W\left(t, u_{m}\right)-\nabla W(t, u)\right|^{2} d t\right)^{\frac{1}{2}}\left\|u_{m}-u\right\| .
\end{aligned}
$$

As in the proof of Lemma 3.1, by passing to a subsequence if necessary, we may assume that $\int_{\mathbb{R}} e^{Q(t)}\left|\nabla W\left(t, u_{m}\right)-\nabla W(t, u)\right|^{2} d t \rightarrow 0$ as $m \rightarrow \infty$. Hence (3.31) implies

$$
\int_{\mathbb{R}} e^{Q(t)}\left(\nabla W\left(t, u_{m}\right)-\nabla W(t, u)\right) \cdot\left(u_{m}-u\right) d t \rightarrow 0 \text { as } m \rightarrow \infty .
$$


Combining (3.28), (3.29), (3.30) and (3.32) yields $u_{m} \rightarrow u$ as $m \rightarrow \infty$ in $E$. The proof is now completed.

Note that (3.24), (3.25) and (3.26) imply

$$
f_{\lambda_{n}}^{\prime}\left(u_{n}^{k}\right)=0, f_{\lambda_{n}}\left(u_{n}^{k}\right) \in\left[\bar{\alpha}_{k}, \bar{\xi}_{k}\right], \forall n \in \mathbb{N} \text { and } k \geq k_{0}
$$

Lemma 3.6. For any $k \geq k_{0}$, the sequence $\left(u_{n}^{k}\right)_{n \in \mathbb{N}}$ obtained above is bounded.

Proof. For notational simplicity, we set $u_{n}=u_{n}^{k}$ for all $n \in \mathbb{N}$. Assuming indirectly that $\left(u_{n}\right)$ is unbounded. By going to a subsequence if necessary, we may assume

$$
\left\|u_{n}\right\| \rightarrow \infty \text { and } v_{n}=\frac{u_{n}}{\left\|u_{n}\right\|} \rightarrow v \text { as } n \rightarrow \infty .
$$

Without loss of generality, Lemma 2.2 and (3.24) imply

$$
v_{n} \rightarrow v \text { both in } L_{Q}^{2}(\mathbb{R}) \text { and } L_{Q}^{v}(\mathbb{R}) \text {, and } v_{n}(t) \rightarrow v(t) \text { a.e. } t \in \mathbb{R} \text { as } n \rightarrow \infty \text {. }
$$

First case: $v=0$. Let $\left(s_{n}\right)$ be a sequence such that

$$
f_{\lambda_{n}}\left(s_{n} u_{n}\right)=\max _{s \in[0,1]} f_{\lambda_{n}}\left(s u_{n}\right), \forall n \in \mathbb{N}
$$

For $R>0$, let $w_{n}=2 \sqrt{R} v_{n}$. By (3.35), we have

$$
w_{n} \rightarrow 2 \sqrt{R} v=0 \text { both in } L_{Q}^{2}(\mathbb{R}) \text { and } L_{Q}^{v}(\mathbb{R}),
$$

which together with $\left(W_{1}\right)$ implies

$$
\left|\int_{\mathbb{R}} e^{Q(t)} W\left(t, w_{n}\right) d t\right| \leq c \int_{\mathbb{R}} e^{Q(t)}\left(\left|w_{n}\right|^{2}+\left|w_{n}\right|^{v}\right) d t \rightarrow 0 \text { as } n \rightarrow \infty .
$$

Note that (3.34) implies that $0<\frac{2 \sqrt{R}}{\left\|u_{n}\right\|}<1$ for $n$ large enough. This together with (3.36) and (3.38) implies

$$
\begin{aligned}
f_{\lambda_{n}}\left(s_{n} u_{n}\right) & \geq f_{\lambda_{n}}\left(w_{n}\right) \\
& =\frac{1}{2}\left\|u_{n}\right\|^{2}-\lambda_{n} \int_{\mathbb{R}} e^{Q(t)} W\left(t, w_{n}\right) d t \\
& \geq 2 R-2 \int_{\mathbb{R}} e^{Q(t)} W\left(t, w_{n}\right) d t \geq R
\end{aligned}
$$

for $n$ large enough. Since $R$ is arbitrarily, it follows that

$$
\lim _{n \rightarrow \infty} f_{\lambda_{n}}\left(s_{n} u_{n}\right)=+\infty
$$

Note that, since $f_{\lambda_{n}}(0)=0$ and $f_{\lambda_{n}}\left(u_{n}\right) \in\left[\bar{\alpha}_{k}, \bar{\xi}_{k}\right]$, then $\left.s_{n} \in\right] 0,1[$ in (3.36) for $n$ large enough. Therefore

$$
0=s_{n} \frac{d}{d s}\left(f_{\lambda_{n}}\right)\left(s u_{n}\right)_{\mid s=s_{n}}=f_{\lambda_{n}}^{\prime}\left(s_{n} u_{n}\right) s_{n} u_{n}
$$


Combining (3.33), (3.39) and $\left(W_{3}\right)$ yields

$$
\begin{aligned}
f_{\lambda_{n}}\left(u_{n}\right)-\frac{1}{2} f_{\lambda_{n}}^{\prime}\left(u_{n}\right) u_{n} & =\frac{\lambda_{n}}{2} \int_{\mathbb{R}} e^{Q(t)} \widehat{W}\left(t, w_{n}\right) d t \\
& \geq \frac{\lambda_{n}}{2 \sigma} \int_{\mathbb{R}} e^{Q(t)} \widehat{W}\left(t, s_{n} w_{n}\right) d t \\
& =\frac{1}{\sigma}\left[f_{\lambda_{n}}\left(s_{n} u_{n}\right)-\frac{1}{2} f_{\lambda_{n}}^{\prime}\left(s_{n} u_{n}\right) s_{n} u_{n}\right] \\
& =\frac{1}{\sigma} f_{\lambda_{n}}\left(s_{n} u_{n}\right) \rightarrow+\infty \text { as } n \rightarrow \infty,
\end{aligned}
$$

a contradiction with (3.33).

Second case: $v \neq 0$. The set $\Lambda=\{t \in \mathbb{R} / v(t) \neq 0\}$ has a positive measure with respect to meas $_{Q}$. By (3.34), it holds that

$$
\left|u_{n}(t)\right| \rightarrow \infty \text { as } n \rightarrow \infty, \forall t \in \Lambda .
$$

Combining (3.35), (3.41) and $\left(W_{2}\right)$, Fatou's lemma implies

$$
\begin{aligned}
\frac{1}{2}-\frac{f_{\lambda_{n}}\left(u_{n}\right)}{\left\|u_{n}\right\|^{2}} & =\lambda_{n} \int_{\mathbb{R}} e^{Q(t)} \frac{W\left(t, u_{n}\right)}{\left\|u_{n}\right\|^{2}} d t \\
& \geq \int_{\Lambda} e^{Q(t)}\left|v_{n}\right|^{2} \frac{W\left(t, u_{n}\right)}{\left|u_{n}\right|^{2}} d t \rightarrow \infty \text { as } n \rightarrow \infty,
\end{aligned}
$$

which provides a contradiction with (3.33) and (3.34). The proof is now completed.

Finally, using the similar arguments in the proof of Lemma 3.5 and in view of Lemma 3.6 and (3.33), we can show that, for any $k \geq k_{0}$, the sequence $\left(u_{n}^{k}\right)_{n \in \mathbb{N}}$ possesses a strong convergent subsequence with the limit $u^{k}$ being a critical point of $f=f_{1}$. Since $\bar{\alpha}_{k} \rightarrow+\infty$ as $k \rightarrow \infty$ and $f\left(u^{k}\right) \in\left[\bar{\alpha}_{k}, \bar{\xi}_{k}\right]$ for all $k \geq k_{0}$, we have that $f$ has infinitely many critical points. Consequently, $(\mathscr{D} \mathscr{V})$ has infinitely many nontrivial fast homoclinic solutions.

\section{REFERENCES}

[1] M. Izydorek, J. Janczewska, Homoclinic solutions for a class of the second order Hamiltonian systems, J. Differential Equations, 219 (2005), 375-389.

[2] J. Jiang, S. Lu, X. Lv, Homoclinic solutions for a class of second order Hamiltonian systems, Nonlinear Anal. 13 (2012), 176-185.

[3] S. Lu, X. Lv, P. Yan, Existence of homoclinics for a class of Hamiltonian systems, Nonlinear Anal. 72 (2010), 390-398.

[4] P.H. Rabinowitz, Homoclinic orbits for a class of Hamiltonian systems, Proc. Roy. Soc. Edinburg 114 A (1990), 33-38.

[5] X.H. Tang, L. Xiao, Homoclinic solutionsd for a class of second order Hamiltonian systems, Nonlinear Anal. 71 (2009), 1140-1152.

[6] R. Yuan, Z. Zhang, Homoclinic solutions for some second order nonautonomous hamiltonian systems without the globally superquadratic condition, Nonlinear Anal. 72 (2010), 1809-1819.

[7] P.H. Rabinowitz, K. Tanaka, Some results on connecting orbits for a class of Hamiltonian systems, Math. Z. 206 (1991), 473-499.

[8] Y. Ding, Existence and multiplicity results for homoclinic solutions to a class of Hamiltonian systems, Nonlinear Anal. 25 (1995), 1095 -1113.

[9] G. Chen, Homoclinic orbits for second order Hamiltonian systems with asymptotically linear terms at infinity, Adv. Difference Equations 2014 (2014), Article ID 114. 
[10] G. Chen, Z. He, Infinitely many homoclinic solutions for a class of second order Hamiltonian systems, Adv. Difference Equations 2014 (2014), 161.

[11] L. Chu, Q. Zhang, Homoclinic solutions for a class of second order Hamiltonian systems with locally defined potentials, Nonlinear Anal. 75 (2012), 3188-3197.

[12] X. Lin, X.H. Tang, Homoclinic solutions for a class of second order Hamiltonian systems, J. Math. Anal. Appl. 354 (2009), 539-549.

[13] X. Lin, X.H. Tang, Infinitely may homoclinic orbits for Hamiltonian systems with indefinite sign subquadratic potentials, Nonlinear Anal. 74 (2011), 6314-6325.

[14] C. Liu, Q. Zhang, Infinitely many homoclinic solutions for second order Hamiltonian systems, Nonlinear Anal. 72 (2010), 894-903.

[15] J. Sun, T-F. Wu, Homoclinic solutions for a second order Hamiltonian system with a positive semi-definite matrix, Chaos, Solitons and Fractals 76 (2015), 24-31.

[16] J. Sun, T-F. Wu, Multiplicity and concentration of homoclinic solutions for some second order Hamiltonian systems, Nonlinear Anal. 114 (2015), 105-115.

[17] C.L. Tang, L.-L. Wan, Existence and homoclinic orbits for second order Hamiltonian systems without (AR) condition, Nonlinear Anal. 74 (2011), 5303-5313.

[18] J. Wei, J. Wang, Infinitely many homoclinic orbits for the second order Hamiltonian systems with general potentials, J. Math. Anal. Appl. 366 (2010), 694-699.

[19] Z. Zhang; Existence of homoclinic solutions for second order Hamiltonian systems with general potentials, J. Appl. Math. Comput. 44 (2014), 263-272.

[20] X.H. Tang, Infinitely many homoclinic solutions for a second-order Hamiltonian system, Math. Nach. 289 (2016), 116-127.

[21] R.P. Agarwal, P. Chen, X. Tang, Fast homoclinic solutions for a class of damped vibration problems, Appl. Math. Comput. 219 (2013), 6053-6065.

[22] P. Chen, X.H. Tang, Fast homoclinic solutions for a class of damped vibration problems with sub-quadratic potentials, Math. Nachr. 286 (2013), 4-16.

[23] M. Timoumi, Infinitely many homoclinic solutions for a class of superquadratic fourth-order differential equations, J. Nonlinear Funct. Anal. 2018 (2018), Article ID 20.

[24] R. Yuan, Z. Zhang, Fast homoclinic solutions for some second order non-autonomous systems, J. Math. Anal. Appl. 376 (2011), 51-63.

[25] W. Zoum Variant fountain theorems and their applications, Manuscripta Math. 104 (2001) 343-358. 